\title{
DEVELOPMENT OF A COUPLED AIR AND PARTICLE THERMAL MODEL FOR ENGINE ICING TEST FACILITIES
}

Tadas Bartkus, Peter Struk, Jen-Ching Tsao

Ohio Aerospace Institute

NASA Glenn Research Center 


\section{Introduction Icing Observations}

- Many engine power-loss events reported since the 1990's

- Mason et al. ${ }^{1}$ hypothesized how power-loss events can result from ice crystals entering the engine core

- Ingestion of ice into engine is studied

- NASA conducted tests at NRC's RATFac

- Observed environmental conditions changed with cloud activation

- Air temperature change

- Air humidity change

- Particle water content change

- Hypothesis : Thermal interaction between air and cloud particles 


\section{Thermal Model General Description}

- Model couples air and cloud particle conservation equations

- Mass, energy and momentum

- Simulates icing tunnel (applicable to engines too)

- Ice, water, and humid air mass broken into fundamental CV units

- Uniform particle size (MVD representation)

- Full particle size distribution

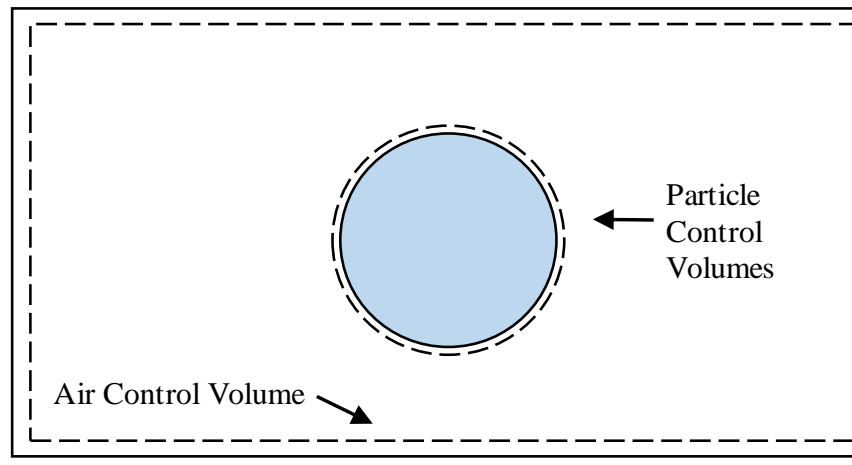

Single particle depiction (uniform particle size cloud)

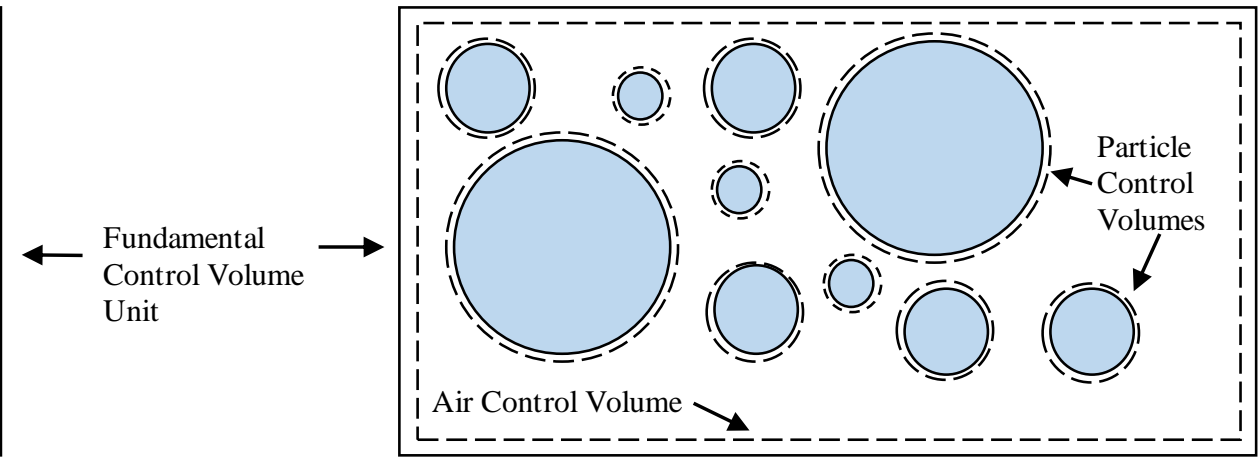

Full particle size distribution particle depiction (multiple particle size cloud) 


\section{Assumptions}

- Air and water vapor are treated as ideal gases

- Air is continually well mixed

- No supersaturation

- 1-D air and particle flow

- Dilute system (no particle interaction)

- Particles are spherical

- Discrete particle size distribution (bins)

- Uniform temperature within the particle

- Supercooling can occur

- Mixed phase particles are not spatially resolved

- Phase change occurs at particle surface at particle temperature

- Adiabatic tunnel walls

- The flow of particles and air is a continuous stream

- The fundamental CV is adiabatic and mass is conserved

- Provisions for heat and mass transfer added using source terms 


\section{Particle Conservation of Energy Single Particle Formulation (1/2)}

- Change in the particle's enthalpy, is due to the convective heat transfer and latent energy exchange due to mass transfer

$$
\frac{\partial H_{p}}{\partial t}=q_{\text {conv }}+q_{\text {latent }}
$$

- Rate of mass change with a water surface is proportional to the difference in vapor pressure between the particle surface and the ambient air

Ice: $\rho_{p} C_{p} \frac{\pi d^{3}}{6} \frac{\partial T_{p}}{\partial t}=\pi d^{2} h\left(T_{\text {air }}-T_{p}\right)+\pi d^{2} h_{m} \rho_{\text {air }} L_{\text {subl }}\left(\omega_{\text {air }}-\omega_{p}\right)$

Mix: $\rho_{p} L_{\text {melt }} \frac{\pi d^{3}}{6} \frac{\partial \eta_{p}}{\partial t}=\pi d^{2} h\left(T_{\text {air }}-T_{p}\right)+\pi d^{2} h_{m} \rho_{\text {air }} L_{\text {subl/evap }}\left(\omega_{\text {air }}-\omega_{p}\right)$

Water: $\rho_{p} C_{p} \frac{\pi d^{3}}{6} \frac{\partial T_{p}}{\partial t}=\pi d^{2} h\left(T_{\text {air }}-T_{p}\right)+\pi d^{2} h_{m} \rho_{\text {air }} L_{\text {evap }}\left(\omega_{\text {air }}-\omega_{p}\right)$ 


\section{Particle Conservation of Energy Single Particle Formulation (2/2)}

- Empirical heat and mass transfer expressions for flow over a sphere ${ }^{2}$

- $N u=\frac{h d}{k_{\text {air }}}=2+0.6 \operatorname{Re}^{1 / 2} \operatorname{Pr}^{1 / 3}$

- $S h=\frac{h_{m} d}{D_{a b}}=2+0.6 R e^{1 / 2} S c^{1 / 3}$

- $R e=\frac{\rho_{\text {air }}\left|v_{\text {air }}-v_{p}\right| d}{\mu_{\text {air }}}$

- $\operatorname{Pr}=\frac{C_{\text {air }} \mu_{\text {air }}}{k_{\text {air }}}$

- $S c=\frac{\mu_{a i r}}{\rho_{a i r} D_{a b}}$

- $\omega_{\text {air }}=\frac{M W_{\text {water }}}{M W_{\text {air }}} \frac{P_{w v, \text { air }}}{P_{\text {air }}}$

- $\omega_{\text {surf }}=\frac{M W_{\text {water }}}{M W_{\text {air }}} \frac{P_{\text {wv,surf }}}{P_{\text {air }}}$ 


\section{Air Conservation of Energy Single Particle Formulation}

- Change in the air enthalpy is due to the convective heat transfer and the sensible energy change of the water vapor mass that has changed phase

$$
\text { - } \frac{\partial H_{\text {air }}}{\partial t}=q_{c o n v}+q_{w v, s e n s}
$$

- $q_{w v, s e n s}$ is thermal mixing

$$
\text { - } m_{\text {air }} C_{\text {air }} \frac{\partial T_{\text {air }}}{\partial t}=\pi d^{2} h\left(T_{p}-T_{\text {air }}\right)+\frac{\partial m_{w v}}{\partial t} \int_{T_{\text {air }}}^{T_{p}} C_{w v} \partial T
$$

- $\mathrm{m}_{\mathrm{wv}}$ can be mass from evaporation or mass to condense 


\section{Conservation of Mass}

\section{Single Particle Formulation}

- Change in particle mass due to vapor phase change

- $\frac{\partial m_{p}}{\partial t}=\rho_{p} \frac{\partial}{\partial t}\left(\frac{\pi d^{3}}{6}\right)=\pi d^{2} h_{m} \rho_{\text {air }}\left(\omega_{\text {air }}-\omega_{p}\right)$

- Change in air mass is opposite the change in particle mass

- $\frac{\partial m_{\text {air }}}{\partial t}=-\frac{\partial m_{p}}{\partial t}=\pi d^{2} h_{m} \rho_{\text {air }}\left(\omega_{p}-\omega_{\text {air }}\right)$ 


\section{Conservation of Energy Particle Distribution Formulation}

- MVD vs particle distribution

- Smaller particles have greater SA/Vol ratio $\rightarrow$ faster transfer response

- Cumulative differences will add up to a different final result

- Fraction of the total water mass calculated for every particle size bin

- An energy balance equation for every particle size $i$

$$
\text { - } \rho_{p, i} C_{p, i} \frac{\pi d_{i}^{3}}{6} \frac{\partial T_{p, i}}{\partial t}=\pi d_{i}^{2} h_{i}\left(T_{\text {air }}-T_{p, i}\right)+\pi d_{i}^{2} h_{m, i} \rho_{\text {air }} L_{i}\left(\omega_{\text {air }}-\omega_{p, i}\right)
$$

- One air energy equation contains the sum of all the particle heat transfers and vapor sensible energy transfers.

- $m_{\text {air }} C_{\text {air }} \frac{\partial T_{\text {air }}}{\partial t}=\sum_{i=1}^{n}\left[\pi d_{i}^{2} h_{i}\left(T_{p, i}-T_{\text {air }}\right)+\frac{\partial m_{w v, i}}{\partial t} \int_{T_{a i r}}^{T_{p, i}} C_{w v, i} \partial T\right]\left(\#_{i}\right)$

- $n=$ number of particle size bins

- $\#_{i}=$ number of particles in the $i^{\text {th }}$ bin 


\section{Conservation of Mass}

\section{Particle Distribution Formulation}

- A mass balance equation for every particle size $i$

$$
\text { - } \frac{\partial m_{p, i}}{\partial t}=\pi d_{i}^{2} h_{m, i} \rho_{a i r}\left(\omega_{a i r}-\omega_{p, i}\right)
$$

- One air mass equation contains the sum of all the particle mass transfers.

$$
\text { - } \frac{\partial m_{\text {air }}}{\partial t}=-\sum_{i=1}^{n}\left[\frac{\partial m_{p, i}}{\partial t}\right]\left(\#_{i}\right)
$$




\section{Conservation of Momentum}

- Conservation of momentum equation is solved in reference to particle

- $F=F_{d r a g}+F_{g}=m_{p} a=\rho_{p} \frac{\pi d^{3}}{6} \frac{\partial v_{p}}{\partial t}$

- $F_{g}=0$

- $F_{\text {drag }}=\frac{1}{2} \rho_{\text {air }} U^{2} A C_{D}$

- $U=v_{\text {air }}-v_{p}$

- $A=\pi d^{2}$

$-C_{D}=\frac{24}{R e}+\frac{2.6\left(\frac{R e}{5.0}\right)}{1+\left(\frac{R e}{5.0}\right)^{1.52}}+\frac{0.411\left(\frac{R e}{263,000}\right)^{-7.94}}{1+\left(\frac{R e}{263,000}\right)^{-8.00}}+\frac{R e^{0.80}}{461,000}$

- $\frac{\partial v_{p}}{\partial t}=\frac{3}{4} \frac{\rho_{a i r} C_{D}\left(v_{a i r}-v_{p}\right)^{2}}{\rho_{p} d}$ 


\section{Supercooled Freezing Formulation}

- Four stages in supercooled freezing

1. Sensible liquid cooling (below 273.15K)

2. Latent heat release

3. Latent freezing (at 273.15K)

4. Sensible ice cooling

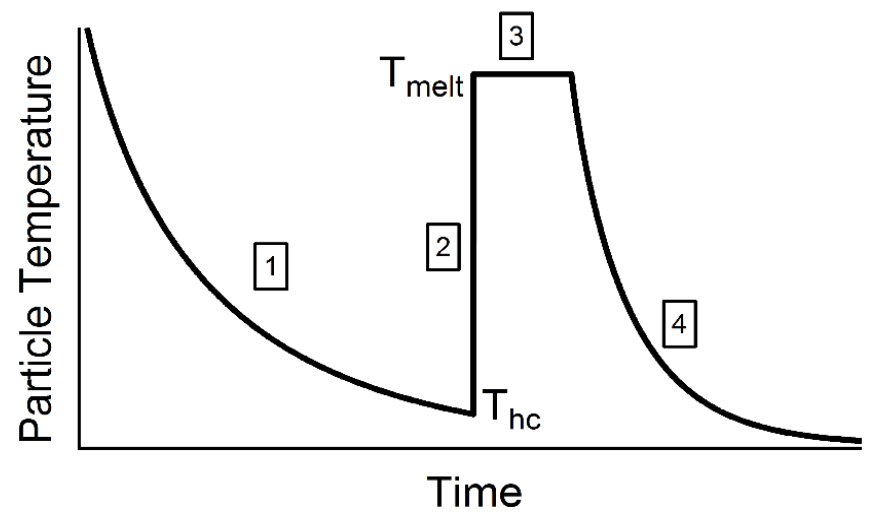

- Homogeneous crystallization temperature as a function of diameter ${ }^{4}$

$-T_{h c}=7.2015 \ln (d)+214.64$

- Expressions to determine melt fraction after latent heat release

- $H_{\text {sens,super }}=m_{p} \int_{T_{h c}}^{273.15} C_{p}(T) d T$

- $\eta_{p}=1-\frac{m_{p} L_{m e l t}-H_{\text {sens,super }}}{m_{p} L_{m e l t}}$ 


\section{Algorithm}

- Written in MATLAB version R2014a

- Solves conservation equations using built-in ODE45 solver

- Relative and absolute convergence tolerance of 10-12

- Mass transferred between the air and particle(s) balanced to $10^{-15}$

- Energy transferred between the air and particle(s) balanced to $10^{-4}$

- Model accuracy dependent on accuracy of property values $\left(C_{p}, L_{\text {heat }}\right.$, etc. $)$ 


\section{Parametric Analysis Baseline 1 Tests}

\begin{tabular}{|l|l|l|}
\hline & Units & Baseline 1 \\
\hline Particle Diameter & $\mu \mathrm{m}$ & 10 \\
\hline LWC & $\mathrm{g} / \mathrm{m}^{3}$ & 1 \\
\hline Pressure & $\mathrm{Pa}$ & 88,000 \\
\hline Relative Humidity & $\%$ & 50 \\
\hline Air Temperature & $\mathrm{K}$ & 278.15 \\
\hline Particle Temperature & $\mathrm{K}$ & 278.15 \\
\hline Slip Velocity & $\mathrm{m} / \mathrm{s}$ & 5
\end{tabular}
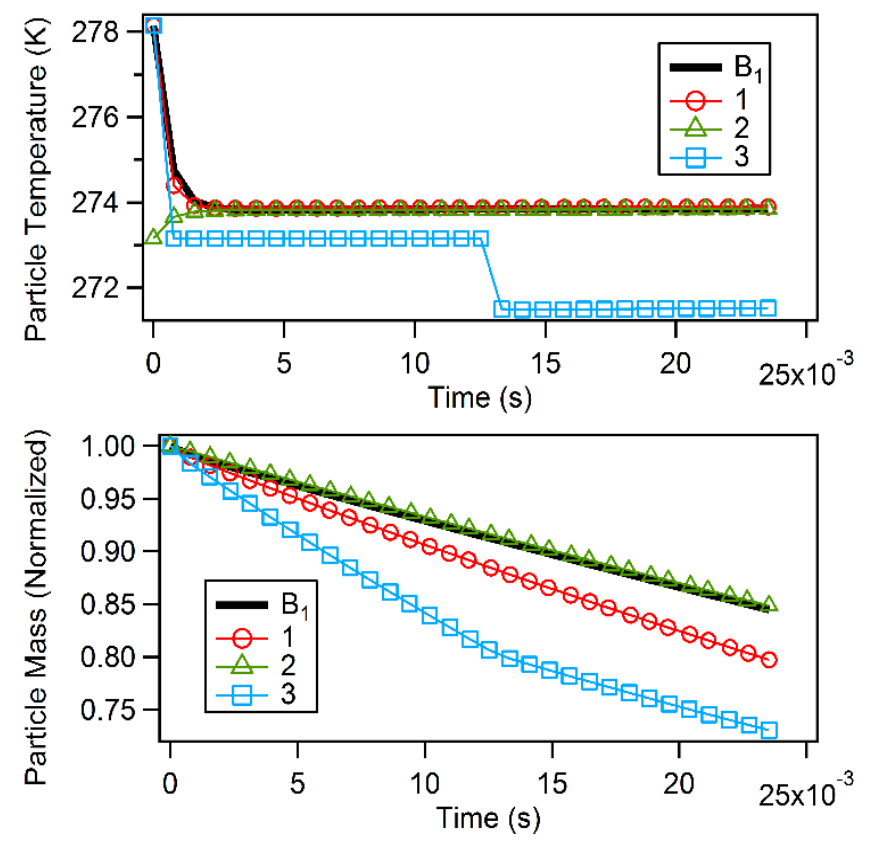

\begin{tabular}{l|l|l|l|}
\hline Test \# & Parameter Changed & Units & Value \\
\hline Baseline 1 & Slip Velocity & $\mathrm{m} / \mathrm{s}$ & 25 \\
\hline $\mathbf{1}$ & Sarticle Temperature & $\mathrm{K}$ & 273.15 \\
\hline $\mathbf{2}$ & Pa & 28,000 \\
\hline $\mathbf{3}$ & Pressure & $\mathrm{Pa}$ & \\
\hline
\end{tabular}

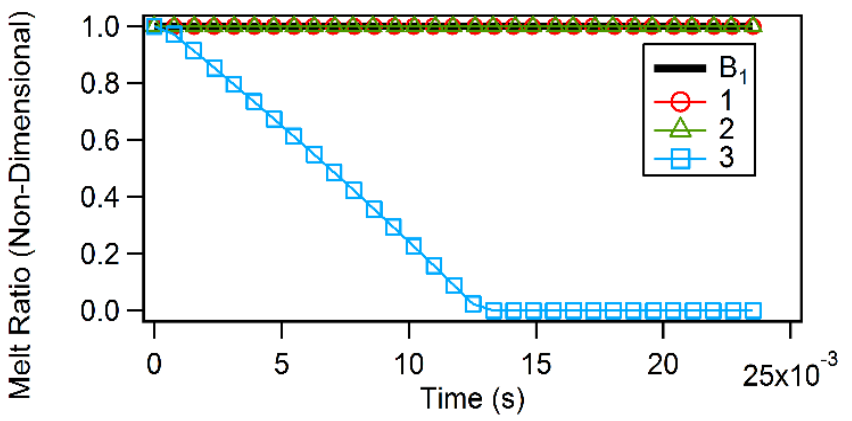

Takeaway: Wet-bulb temperature determines state of particle 


\section{Parametric Analysis Baseline 2 Tests}

\begin{tabular}{|l|l|l|}
\hline & Units & Baseline 2 \\
\hline Particle Diameter & $\mu \mathrm{m}$ & 10 \\
\hline IWC & $\mathrm{g} / \mathrm{m}^{3}$ & 1 \\
\hline Pressure & $\mathrm{Pa}$ & 88,000 \\
\hline Relative Humidity & $\%$ & 50 \\
\hline Air Temperature & $\mathrm{K}$ & 280.15 \\
\hline Particle Temperature & $\mathrm{K}$ & 271.15 \\
\hline Slip Velocity & $\mathrm{m} / \mathrm{s}$ & 5 \\
\hline
\end{tabular}

\begin{tabular}{l|l|l|l|}
\hline \begin{tabular}{l} 
Test \# \\
\hline Baseline 2
\end{tabular} & Parameter Changed & Units & Value \\
\hline $\mathbf{4}$ & IWC & $\mathrm{g} / \mathrm{m}^{3}$ & 5 \\
\hline $\mathbf{5}$ & Relative Humidity & $\%$ & 80 \\
\hline $\mathbf{6}$ & Air Temperature & $\mathrm{K}$ & 271.15 \\
\hline
\end{tabular}
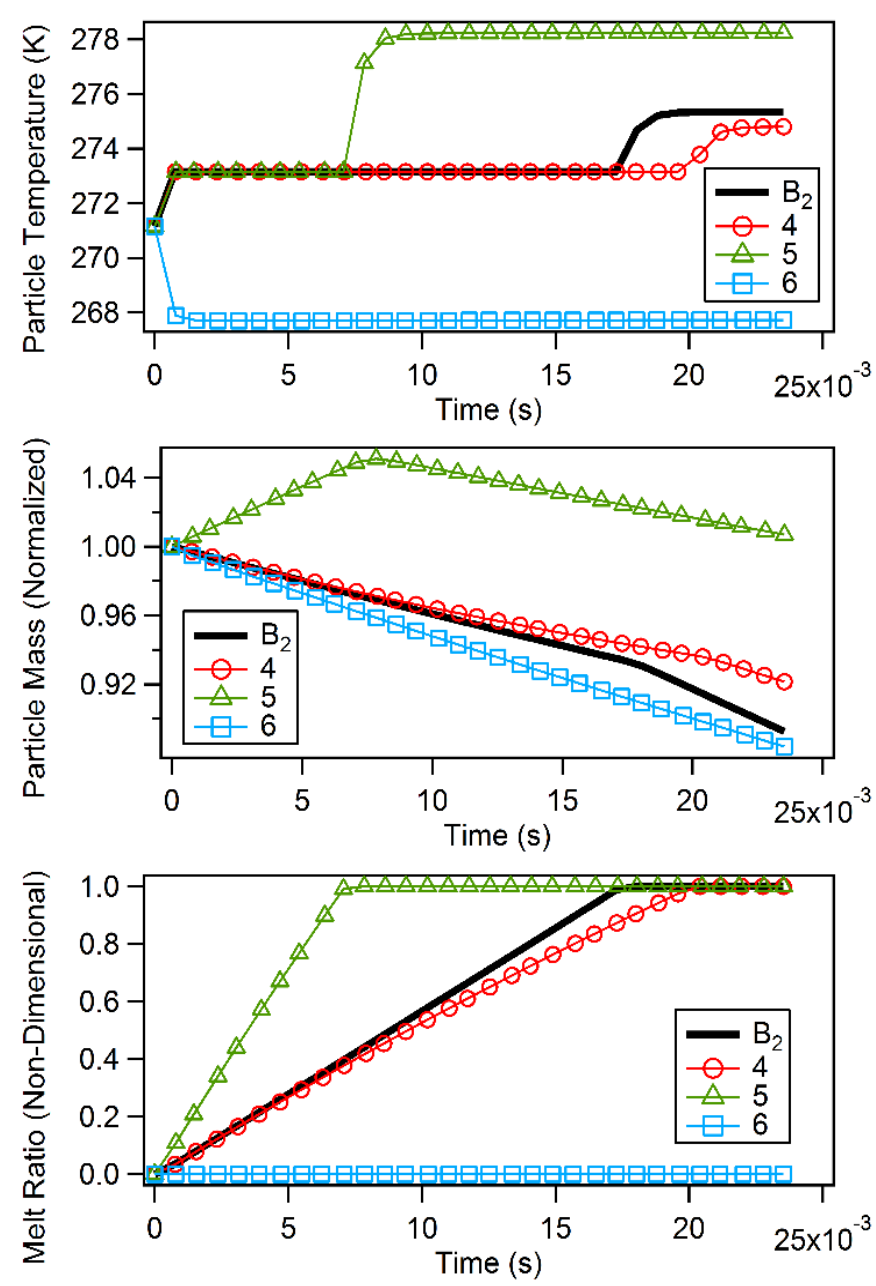

Takeaway: Air thermal mass $>>$ Particle thermal mass 
- Air velocity, air temperature, and vapor content vary from injection to test section at RATFac (no icing cloud)

- CFD model previously written to approximate $\mathrm{v}_{\text {air }}$ and $\mathrm{T}_{\mathrm{air}}$

- Centerline values normalized

- Water vapor profile analogous to normalized air temperature profile

- Approximate changing values as sources in expressions (no cloud)

- Valid for tests Mach $\sim 0.2$ and $\mathrm{P}_{\text {total }} \sim 44000 \mathrm{~Pa}$ at test section

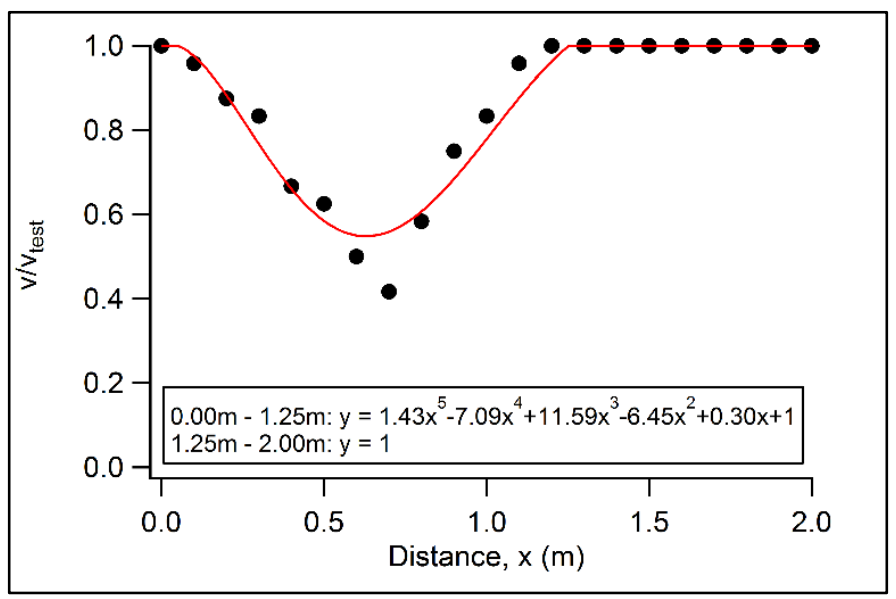

Normalized air velocity at centerline (without an icing cloud)

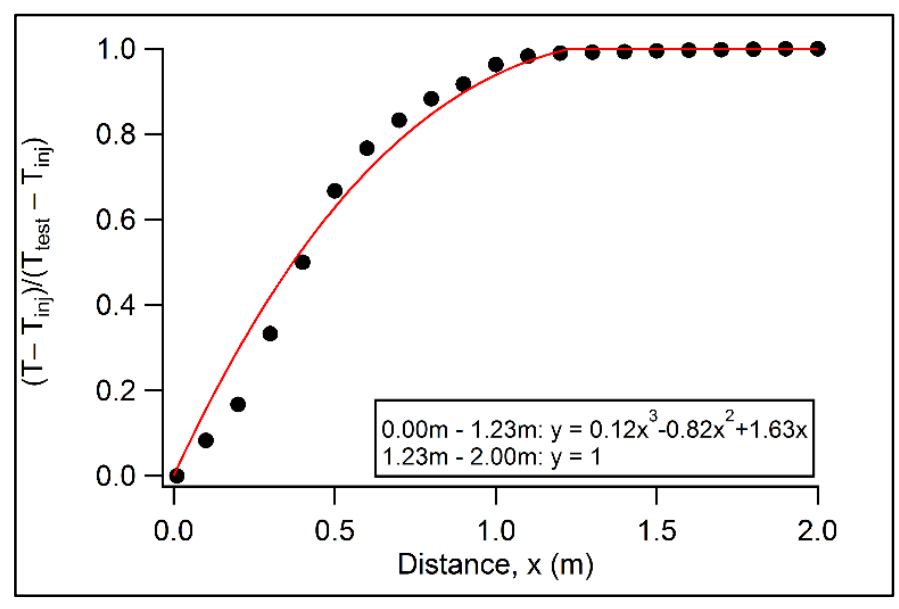

Normalized air temperature at centerline (without an icing cloud) 


\section{Model - Experiment Comparison}

\begin{tabular}{|c|c|c|c|c|}
\hline & Units & Scan 877 & Scan 983 & Scan 1003 \\
\hline$T_{\text {air,inj }}$ & $\mathrm{K}$ & 262.0 & 256.1 & 256.9 \\
\hline $\mathbf{T}_{\text {air, target }}$ & $\mathrm{K}$ & 288.4 & 278.0 & 277.9 \\
\hline $\begin{array}{l}\mathrm{SH}_{\mathrm{inj}} \\
\left(\mathrm{RH}_{\mathrm{inj}}\right)\end{array}$ & $\begin{array}{l}\mathrm{g}_{\mathrm{vapor}} / \mathrm{kg}_{\mathrm{dry} \text { air }} \\
(\%)\end{array}$ & $\begin{array}{l}0.07 \\
(2)\end{array}$ & $\begin{array}{l}0.07 \\
(5)\end{array}$ & $\begin{array}{l}0.07 \\
(4)\end{array}$ \\
\hline $\begin{array}{l}\mathrm{SH}_{\text {target }} \\
\left(\mathrm{RH}_{\text {target }}\right)\end{array}$ & $\begin{array}{l}\mathrm{g}_{\mathrm{vapor}} / \mathrm{kg}_{\mathrm{dry} \text { air }} \\
(\%)\end{array}$ & $\begin{array}{l}4.07 \\
(16.1)\end{array}$ & $\begin{array}{l}2.88 \\
(35.4)\end{array}$ & $\begin{array}{l}3.81 \\
(46.5)\end{array}$ \\
\hline GWC $_{\text {inj/target }}$ & $\mathrm{g} / \mathrm{m}^{3}$ & 2.1 & 2.4 & 3.0 \\
\hline LWC $_{\text {inj/target }}$ & $\mathrm{g} / \mathrm{m}^{3}$ & 1.0 & 0 & 1.9 \\
\hline$I_{W} C_{\text {inj/target }}$ & $\mathrm{g} / \mathrm{m}^{3}$ & 0 & 8.4 & 8.6 \\
\hline$\eta_{\text {inj/target }}$ & - & 1.0 & 0.0 & 0.18 \\
\hline $\mathbf{P}$ & $\mathrm{Pa}$ & 42806 & 66478 & 65934 \\
\hline $\mathbf{V}_{\text {air,inj/target }}$ & $\mathrm{m} / \mathrm{s}$ & 86.8 & 85.7 & 84.1 \\
\hline $\mathrm{T}_{\text {water,inj }}$ & $\mathrm{K}$ & 278.15 & - & 278.15 \\
\hline$T_{\text {ice,inj }}$ & $\mathrm{K}$ & - & 256.15 & 256.15 \\
\hline MVD $_{\text {water,inj }}$ & $\mu \mathrm{m}$ & 40.0 & - & 40.0 \\
\hline$M_{\text {ice,inj }}$ & $\mu \mathrm{m}$ & - & 45.5 & 45.5 \\
\hline
\end{tabular}

\begin{tabular}{|c|c|c|c|c|}
\hline & Units & Scan 877 & Scan 983 & Scan 1003 \\
\hline$\Delta T_{\text {air, }} \exp$ & K & -2.6 & -2.9 & -4.4 \\
\hline$\Delta T_{\text {air }, \text { sim }}$ & $\mathrm{K}$ & -0.54 & -0.75 & -0.88 \\
\hline$\Delta G W C_{\exp }$ & $\mathrm{g} / \mathrm{m}^{3}$ & 0.5 & 1.0 & 1.2 \\
\hline$\Delta G^{\prime} C_{\text {sim }}$ & $\mathrm{g} / \mathrm{m}^{3}$ & 0.13 & 0.16 & 0.21 \\
\hline$\Delta L W C_{\exp }$ & $\mathrm{g} / \mathrm{m}^{3}$ & -0.5 & 0.5 & 0.2 \\
\hline$\Delta \mathrm{LWC}_{\text {sim }}$ & $\mathrm{g} / \mathrm{m}^{3}$ & -0.13 & 0.0 & -0.09 \\
\hline$\Delta \mathrm{IWC}_{\exp }$ & $\mathrm{g} / \mathrm{m}^{3}$ & 0.0 & -1.5 & -1.4 \\
\hline$\Delta \mathrm{IWC}_{\text {sim }}$ & $\mathrm{g} / \mathrm{m}^{3}$ & 0.0 & -0.16 & -.12 \\
\hline$\Delta \eta_{\exp }$ & - & 0.00 & 0.07 & 0.05 \\
\hline$\Delta \eta_{\text {sim }}$ & - & 0.00 & 0.00 & -0.004 \\
\hline
\end{tabular}

- Conditions

- Scan 887: Water sprayed into low $\mathrm{P}$, low $\mathrm{RH}$, very warm air

- Scan 983: Ice sprayed into medium $\mathrm{P}$, medium $\mathrm{RH}$, warm air

- Scan 1003: Water \& Ice sprayed into medium $\mathrm{P}$, medium $\mathrm{RH}$, warm air

Takeaway: Model accounts for $\sim 20 \%$ of experimentally observed changes 


\section{Sources for Model - Experiment Discrepancy}

- Experimentally observed water/ice film on tunnel walls

- Increased residence time for evaporation

- Poor approximation for the vapor mass source term

- Non-spherical particles increase heat and mass transfer

- Turbulence at spray nozzle may enhance transfer

- Uncertainties with experimentally measured values

- Independent corroborating measurements minimize this possibility 


\section{Conclusion}

\section{Thermal model that couples particle and air conservation equations}

- Simulates:

- All phase change types

- Supercooled and normal freezing

- Single particle and full particle distribution sprays (ice, water, combined)

- Complicated icing tunnels with energy and mass sources

- Air temperature, pressure (air mass), and $\mathrm{RH}$ dominate $\rightarrow \mathrm{T}_{\mathrm{wb}}$

- Model compared to experiments conducted at NRC

- Simulated $\sim 20 \%$ of the cloud and air changes observed experimentally

- Reasons for discrepancy are offered

- Future work to determine sources for discrepancy

- Model can be modified to simulate other icing facilities (PSL) 